\title{
A New Alpha Power Weibull Model for Analyzing Time-to-Event Data: A Case Study from Football
}

\author{
Gao Shengjie, ${ }^{1}$ Alisa Craig, ${ }^{2}$ and Getachew Tekle Mekiso ${ }^{3}{ }^{3}$ \\ ${ }^{1}$ China Football College, Beijing Sport University, Beijing, China \\ ${ }^{2}$ Department of Statistics, Pennsylvania State University, University Park, PA, USA \\ ${ }^{3}$ Department of Statistics, Wachemo University, Hossana, Ethiopia \\ Correspondence should be addressed to Getachew Tekle Mekiso; getch.55tekle@gmail.com
}

Received 28 November 2021; Revised 21 December 2021; Accepted 21 January 2022; Published 9 February 2022

Academic Editor: Saima K Khosa

Copyright ( $\odot 2022$ Gao Shengjie et al. This is an open access article distributed under the Creative Commons Attribution License, which permits unrestricted use, distribution, and reproduction in any medium, provided the original work is properly cited.

\begin{abstract}
Statistical methodologies have wider applications in exercise science, sports medicine, sports management, sports marketing, sports science, and other related sciences. These methods can be used to predict the winning probability of a team or individual in a match, the number of minutes that an individual player will spend on the ground, the number of goals to be scored by an individual player, the number of red/yellow cards that will be issued to an individual player or a team, etc. Keeping in view the importance and applicability of the statistical methodologies in sport sciences, healthcare, and other related sectors, this paper introduces a novel family of statistical models called new alpha power family of distributions. It is shown that numerous properties of the suggested method are similar to those of the new Weibull- $X$ and exponential type distributions. Based on the novel method, a special model, namely, a new alpha power Weibull distribution, is studied. The new model is very flexible because the shape of its probability density function can either be right-skewed, decreasing, left-skewed, or increasing. Furthermore, the new distribution is also able to model real phenomena with bathtub-shaped failure rates. Finally, the usefulness/applicability of the proposed distribution is shown by analyzing the time-to-event datasets selected from different football matches during $1964-2018$.
\end{abstract}

\section{Introduction}

In the practice of healthcare [1], reliability [2], education [3], hydrology [4], management [5], metrology [6], and sports sciences [7], statistical modeling and predicting real-life events are very crucial. Numerous statistical models (traditional/classical and modified distributions) have been suggested to model data in these sectors.

In the recent era, the development of generating new families of statistical models has received considerable attention [8]. Recently, Alzaatreh et al. [9] introduced an interesting method, namely, the $T-X$ approach, to update the distributional flexibility of the existing models. The DF (distribution function) $K(y ; \Phi, \Xi)$ of the $T$-X method is given by

$$
K(y ; \Phi, \Xi)=\int_{a}^{F[G(y ; \Phi)]} v(t ; \Gamma) d t
$$

where the function $F[G(y ; \Phi)]$ fulfills some certain conditions (for details, see [9]). In equation (1), $G(y ; \Phi)$ is a baseline DF with parameter vector $\Phi$ and $v(t ; \Xi)$ is the probability density function (PDF) of the parent model with parameter vector $\Phi$. Corresponding to $K(y ; \Phi, \Xi)$, the PDF $k(y ; \Phi, \Xi)$ is given by

$$
k(y ; \boldsymbol{\Phi}, \boldsymbol{\Gamma})=\left\{\frac{d}{d y} F[G(y ; \boldsymbol{\Phi})]\right\} r\{F[G(y ; \boldsymbol{\Phi})]\} .
$$

Most of the exponential type distributions belong to the class defined in equation (1). Some implemented functions of $F[G(y ; \Phi)]$ are provided in Table 1 .

Recently, Ahmad et al. [19] implemented the T-X approach and introduced an interesting method, namely, a new Weibull- $X$ (NWei- $X$ ) family, to update the distributional flexibility of the classical or modified distributions. They introduced the NWei- $X$ family by using $F[G(y ; \Phi)]=$ $-\log (1-G(y ; \Phi)) / 1-G(y ; \Phi) \quad$ in equation (1) with 
Table 1: Some used functions of $F[G(y ; \Phi)]$ via the $T-X$ method.

\begin{tabular}{lccc}
\hline S. no. & $F[G(y ; \Phi)]$ & Range of $T$ & Members of $T$ - $X$ family \\
\hline 1 & $G(y ; \Phi)$ & {$[0,1]$} & {$[10]$} \\
2 & $-\log (G(y ; \Phi))$ & $(0, \infty)$ & {$[11]$} \\
3 & $-\log [1-G(y ; \Phi)]$ & $(0, \infty)$ & {$[12]$} \\
4 & $-\log \left[1-G^{\alpha}(y ; \Phi)\right]$ & $(0, \infty)$ & {$[13]$} \\
5 & $G(y ; \Phi) / 1-G(y ; \Phi)$ & $(0, \infty)$ & {$[14]$} \\
6 & $-\log \left(1-G(y ; \Phi) / e^{G(y ; \Phi)}\right.$ & $(0, \infty)$ & {$[15]$} \\
7 & $-\log \left(1-G(y ; \Phi) / \beta^{G(y ; \Phi)}\right)$ & {$[16]$} \\
8 & $-\log \left(1-G^{\alpha}(y ; \Phi) / e^{G^{\alpha}(y ; \Phi)}\right.$ & $(0, \infty)$ & {$[17]$} \\
9 & $-\log \left(\sigma\left(1-G^{\alpha}(y ; \Phi)\right) / \sigma-G^{\alpha}(y ; \Phi)\right)$ & $(0, \infty)$ & $[19])(\mathrm{implemented)}$ \\
10 & $-\log (1-G(y ; \Phi)) / 1-G(y ; \Phi)$ & $(-\infty, \infty)$ & {$[20]$} \\
11 & $-\log (G(y ; \Phi) / 1-G(y ; \Phi))$ & $(-\infty, \infty)$ & {$[21]$} \\
12 & $\log (-\log [1-G(y ; \Phi)])$ & & \\
\hline
\end{tabular}

$v(t ; \Xi)=\alpha t^{\alpha-1} e^{-t^{\alpha}}$, where $v(t ; \Xi)$ is the PDF of the oneparameter Weibull model with parameter vector $\Xi=\alpha$. The DF of the NWei- $X$ family is given by

$$
\begin{array}{r}
K(y ; \Delta)=1-\exp \left\{-\left(\frac{-\log (1-G(y ; \boldsymbol{\Phi}))}{1-G(y ; \boldsymbol{\Phi})}\right)^{\alpha}\right\}, \\
\alpha>0, y \in \mathbb{R},
\end{array}
$$

where $\Delta$ is a parameter vector. The expression $K(y ; \Delta)$ can also be written as

$$
\begin{array}{r}
K(y ; \Delta)=1-\exp \left\{-\left(\frac{H(y ; \Phi)}{1-G(y ; \boldsymbol{\Phi})}\right)^{\alpha}\right\}, \\
\alpha>0, y \in \mathbb{R},
\end{array}
$$

where $H(y ; \Phi)$ is the $\mathrm{CHF}$ (cumulative hazard function) associated with DF $G(y ; \Phi)$.

In terms of CHF $M(y ; \Delta)$, the DF $W(y ; \Delta)$ of the exponential type distributions is defined as

$$
\begin{aligned}
W(y ; \Delta) & =1-e^{-M(y ; \Delta)}, \\
y & \in \mathbb{R},
\end{aligned}
$$

where $M(y ; \Delta)$ must fulfil certain conditions as given below:

(i) $M(y ; \Delta)$ is a nonnegative, differentiable, and increasing function of $y$.

(ii) $\lim _{y \longrightarrow-\infty} M(y ; \Delta) \longrightarrow 0$, and $\lim _{y \longrightarrow \infty} M(y ; \Delta)$ $\longrightarrow \infty$.

The traditional exponential, Rayleigh, Weibull, and other extended lifetime distributions belong to the class defined in equation (5) (see [22]). In link to equation (5), the PDF $w(y ; \Delta)$ is given by

$$
\begin{aligned}
w(y ; \Delta) & =m(y ; \Delta) e^{-M(y ; \Delta)}, \\
y & \in \mathbb{R},
\end{aligned}
$$

where $d / d y M(y ; \Delta)=m(y ; \Delta)$.
Here, we introduce an additional parameter in equation (5), by replacing the exponent term with $\alpha$ to propose a very flexible family by the DF

$$
\begin{aligned}
W(y ; \Delta) & =1-\alpha^{-M(y ; \Delta)}, \\
\alpha & >1, \alpha \neq e, y \in \mathbb{R} .
\end{aligned}
$$

For $\alpha=e$, the DF in equation (7) becomes similar to equation (5). The function $M(y ; \Delta)$ fulfills the conditions given in (i) and (ii). As it is quite clear that

$$
0 \leq W(y ; \Delta) \leq 1,
$$

for clarification, let

$$
\begin{gathered}
\lim _{y \longrightarrow-\infty} W(y ; \Delta)=\lim _{y \longrightarrow-\infty}\left(1-\alpha^{-M(y ; \Delta)}\right)=0, \\
\lim _{y \longrightarrow \infty} W(y ; \Delta)=\lim _{y \longrightarrow \infty}\left(1-\alpha^{-M(y ; \Delta)}\right)=1 .
\end{gathered}
$$

Hence, using the results obtained in equations (9) and (10), we observed that the function $W(y ; \Delta)$ defined in equation (7) is a proper DF. The expression in equation (7) is very interesting and can be useful to generate new statistical models belonging to the $T-X$ family of distributions.

Now, we introduce the proposed family called new alpha power (NAPow) family by using $W(y ; \Delta)=-\log (1-G(y ; \Phi)) / 1-G(y ; \Phi)$ in equation (7). The DF of the NAPow family is given by

$W(y ; \boldsymbol{\Delta})=1-\alpha^{-(-\log (1-G(y ; \boldsymbol{\Phi})) / 1-G(y ; \boldsymbol{\Phi}))}, \alpha>1, \alpha \neq e, y \in \mathbb{R}$.

The DF in equation (11) can also be written as

$$
W(y ; \boldsymbol{\Delta})=1-\alpha^{-(H(y ; \boldsymbol{\Phi}) / 1-G(y ; \boldsymbol{\Phi}))}, \alpha>1, \alpha \neq e, y \in \mathbb{R} .
$$

Corresponding to $W(y ; \Delta)$, the PDF $w(y ; \Delta)$, SF (survival function) $S(y ; \Delta)=1-W(y ; \Delta)$, and HF (hazard function) $h(y ; \Delta)=w(y ; \Delta) / 1-W(y ; \Delta)$ are given by 

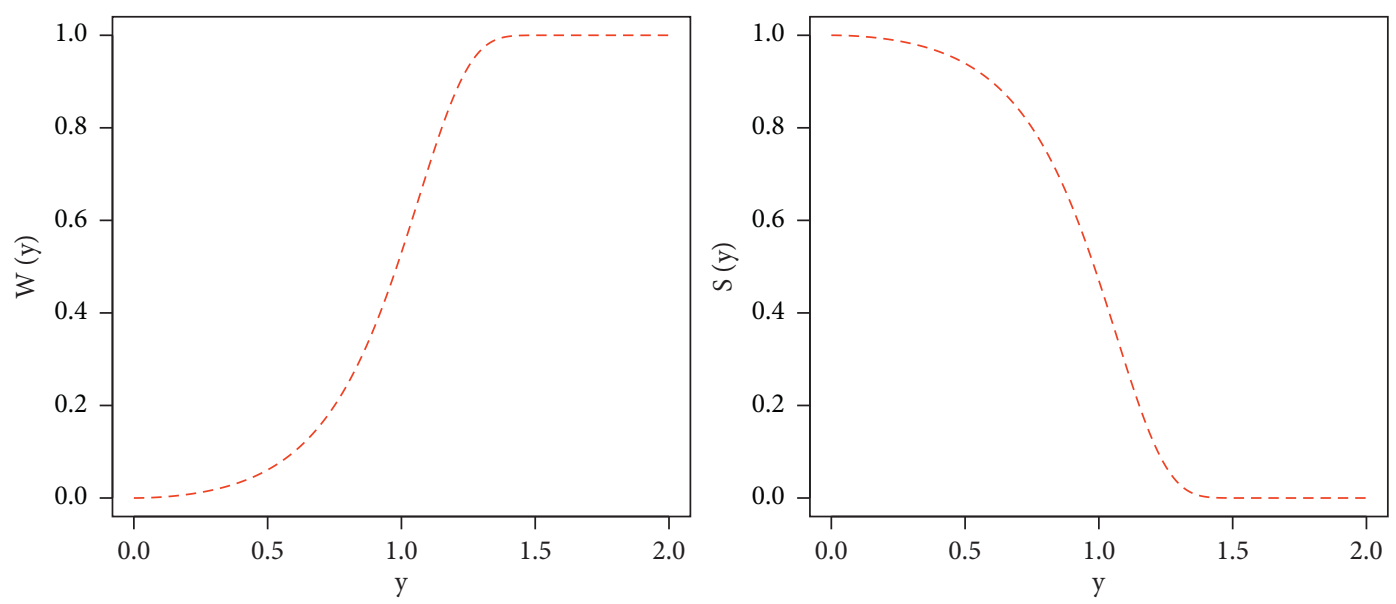

Figure 1: A visual display of $W(y ; \Delta)$ and $S(y ; \Delta)$.

$$
\begin{aligned}
& w(y ; \Delta)=\frac{(\log \alpha) g(y ; \Phi)[1+H(y ; \Phi)]}{[1-G(y ; \boldsymbol{\Phi})]^{2}} \alpha^{-(H(y ; \Phi) / 1-G(y ; \Phi))}, \alpha>1, \alpha \neq e, y \in \mathbb{R}, \\
& S(y ; \Delta)=\alpha^{-(H(y ; \Phi) / 1-G(y ; \Phi))}, \alpha>1, \alpha \neq e, y \in \mathbb{R}, \\
& h(y ; \Delta)=\frac{(\log \alpha) g(y ; \Phi)[1+H(y ; \boldsymbol{\Phi})]}{[1-G(y ; \boldsymbol{\Phi})]^{2}}, \alpha>1, \alpha \neq e, y \in \mathbb{R},
\end{aligned}
$$

respectively.

Due to an extra parameter in the place of the exponent term in equation (7), the NAPow family delivers greater flexibility.

\section{A New Alpha Power Weibull Model}

Here, we discuss a special member of the NAPow family called new alpha power Weibull (NAPow-Weibull) model. The NAPow-Weibull model is introduced by using the DF of the Weibull (two-parameter) distribution as a parent model. A random variable $Y$ has the Weibull model, if its DF $G(y ; \Phi)$ is given by

$$
\begin{aligned}
G(y ; \Phi) & =1-e^{-\varphi y^{\phi}}, \\
y & \geq 0, \phi>0, \varphi>0,
\end{aligned}
$$

where $\Phi=(\phi, \varphi)$. Corresponding to $G(y ; \Phi)$, the $\operatorname{PDF}$ $g(y ; \Phi)$ is given by

$$
\begin{aligned}
g(y ; \Phi) & =\phi \varphi y^{\phi-1} e^{-\varphi y^{\phi}} \\
y & >0, \phi>0, \varphi>0
\end{aligned}
$$

Corresponding to $G(y ; \Phi)$ and $g(y ; \Phi)$, the $\operatorname{HF} h(y ; \Phi)$ and $\mathrm{CHF} H(y ; \Phi)$ are given by

$$
\begin{aligned}
h(y ; \Phi) & =\phi \varphi y^{\phi-1}, \\
y & >0, \phi>0, \varphi>0, \\
H(y ; \boldsymbol{\Phi}) & =\varphi y^{\phi}, y>0, \phi>0, \varphi>0,
\end{aligned}
$$

respectively.

Using $H(y ; \Phi)=\varphi y^{\phi}$ and $G(y ; \Phi)=1-e^{-\varphi y^{\phi}}$ in equation (12), we get the $\operatorname{DF} W(y ; \Delta)$ of the NAPowWeibull model, given by

$$
\begin{aligned}
& W(y ; \Delta)=1-\alpha^{-\varphi y^{\phi} e^{\varphi y^{\phi}}}, \\
& y \geq 0, \phi>0, \varphi>0, \alpha>1, \alpha \neq e,
\end{aligned}
$$

where $\Delta=(\alpha, \phi, \varphi)$.

Corresponding to $W(y ; \Delta)$, the $\operatorname{SF} S(y ; \Delta)$ is given by

$$
\begin{aligned}
& S(y ; \Delta)=\alpha^{-\varphi y^{\phi} e^{\varphi y^{\phi}}}, \\
& y>0, \phi>0, \varphi>0, \alpha>1, \alpha \neq e .
\end{aligned}
$$

A visual display of $W(y ; \Delta)$ and $S(y ; \Delta)$ for $\alpha=1.1, \varphi=1.6$, and $\phi=1.9$ is presented in Figure 1 .

In link to $W(y ; \Delta)$, the PDF $w(y ; \Delta)$ is given by

$$
\begin{aligned}
& w(y ; \Delta)=(\log \alpha) \phi \varphi y^{\phi-1}\left(1+\varphi y^{\phi}\right) \alpha^{-\varphi y^{\phi} e^{\varphi y^{\phi}}}, \\
& y>0, \phi>0, \varphi>0, \alpha>1, \alpha \neq e .
\end{aligned}
$$

In Figure 2, a visual illustration of $w(y ; \Delta)$ is presented for (i) $\alpha=1.2, \varphi=0.9, \phi=1.5$ (red line), (ii) $\alpha=1.9, \varphi=$ $0.9, \phi=0.7$ (green line), (iii) $\alpha=1.1, \varphi=0.9, \phi=2.1$ (blue line), and (iv) $\alpha=1.1, \varphi=0.1, \phi=4.1$ (gold line). by

Furthermore, in link to $W(y ; \Delta)$, the $\operatorname{HF} h(y ; \Delta)$ is given

$$
\begin{array}{r}
h(y ; \Delta)=(\log \alpha) \phi \varphi y^{\phi-1}\left(1+\varphi y^{\phi}\right), \\
y>0, \phi>0, \varphi>0, \alpha>1, \alpha \neq e .
\end{array}
$$



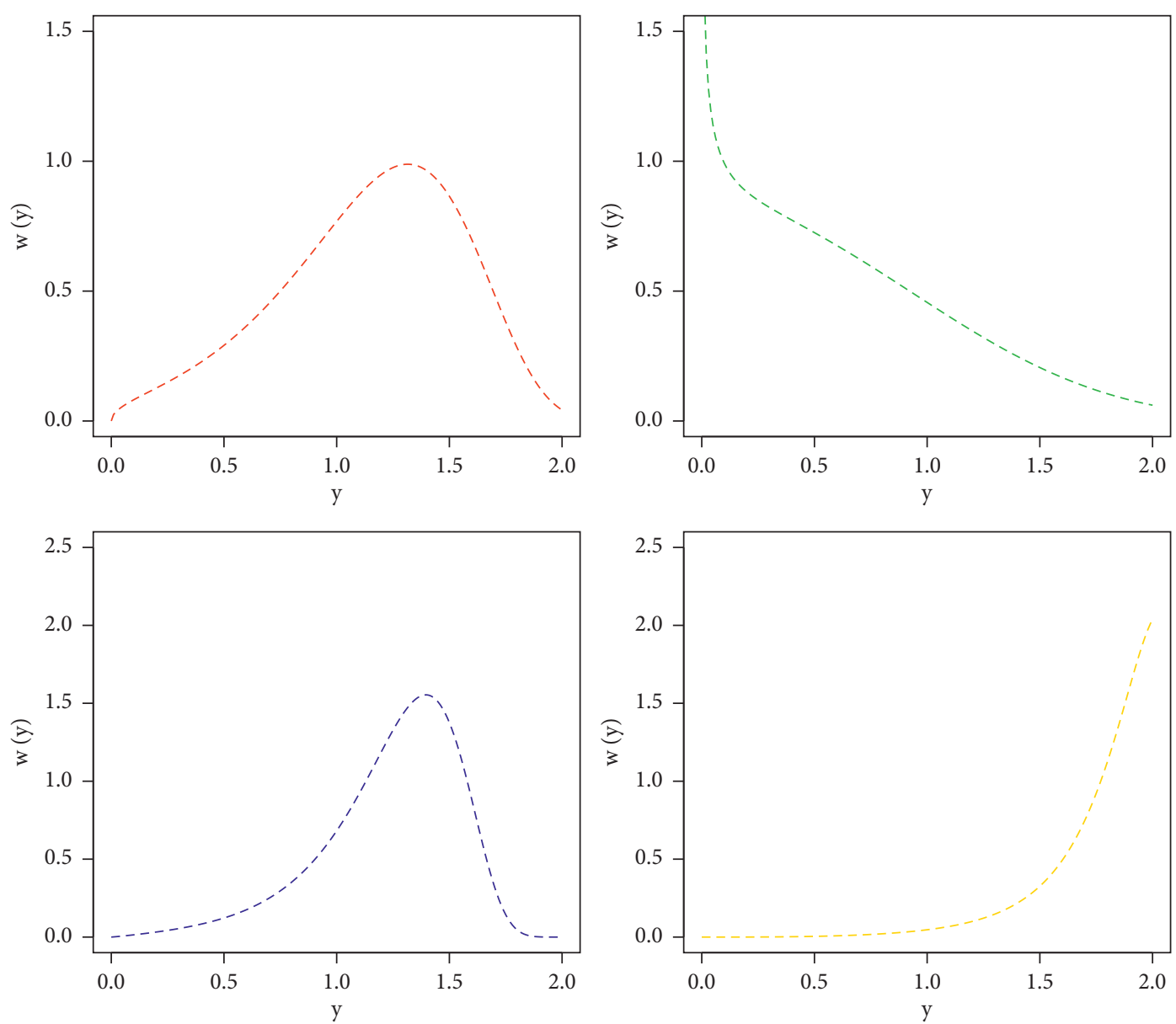

Figure 2: A visual illustration of $w(y ; \Delta)$ for different values of $\alpha, \varphi$, and $\phi$.

Some graphical sketches of $h(y ; \Delta)$ are provided in Figure 3. The plots of $h(y ; \Delta)$ in Figure 3 are obtained for (i) $\alpha=1.5, \varphi=0.1, \phi=3.8$ (blue line), (ii) $\alpha=2.5, \varphi=0.7, \phi=$ 0.08 (green line), and (iii) $\alpha=9, \varphi=0.4, \phi=0.8$ (red line).

\section{Estimation}

Here, we derive the estimators $(\widehat{\alpha}, \widehat{\varphi}, \widehat{\phi})$ of the parameter $(\alpha, \varphi, \phi)$ of the NAPow-Weibull model with PDF $w(y ; \Delta)$. Consider a RS (random sample) say $Y_{1}, Y_{2}, \ldots, Y_{p}$ from PDF $w(y ; \Delta)$. Then, corresponding to $w(y ; \Delta)$, the log likelihood function $\lambda(\Delta)$ is

$$
\begin{aligned}
\lambda(\Delta)= & p \log (\log \alpha)+p \log \phi+p \log \varphi+(\phi-1) \sum_{v=1}^{p} \log y_{v} \\
& +\varphi \sum_{v=1}^{p} y_{v}^{\phi}+\sum_{v=1}^{p} \log \left(1+\varphi y_{v}^{\phi}\right) \\
& -\varphi \sum_{v=1}^{p} y_{v}^{\phi} e^{\varphi y_{v}^{\phi}}(\log \alpha) .
\end{aligned}
$$

The partial derivatives of $\lambda(\Delta)$ are given by

$$
\begin{aligned}
\frac{\partial}{\partial \alpha} \lambda(\Delta)= & \frac{p}{(\log \alpha) \alpha}-\frac{\varphi}{\alpha} \sum_{v=1}^{p} y_{v}^{\phi} e^{\varphi y_{v}^{\phi}} \\
\frac{\partial}{\partial \varphi} \lambda(\Delta)= & \frac{p}{\varphi}+\sum_{v=1}^{p} y_{v}^{\phi}+\sum_{v=1}^{p} \frac{y_{v}^{\phi}}{\left(1+\varphi y_{v}^{\phi}\right)} \\
& -(\log \alpha) \sum_{v=1}^{p} y_{v}^{\phi} e^{\varphi y_{v}^{\phi}}\left[1+\varphi y_{v}^{\phi}\right]
\end{aligned}
$$

$$
\begin{aligned}
\frac{\partial}{\partial \phi} \lambda(\Delta)= & \frac{p}{\phi}+\sum_{v=1}^{p} \log y_{v}+\varphi \sum_{v=1}^{p}\left(\log y_{v}\right) y_{v}^{\phi}+\varphi \sum_{v=1}^{p} \frac{\left(\log y_{v}\right) y_{v}^{\phi}}{\left(1+\varphi y_{v}^{\phi}\right)} \\
& -(\log \alpha) \varphi \sum_{v=1}^{p}\left(\log y_{v}\right) y_{v}^{\phi} e^{\varphi y_{v}^{\phi}}\left(1+\varphi y_{v}^{\phi}\right)
\end{aligned}
$$

respectively.

The estimators of $\alpha, \varphi$, and $\phi$ can be obtained by solving $\partial / \partial \alpha \lambda(\Delta)=0, \partial / \partial \varphi \lambda(\Delta)=0$, and $\partial / \partial \phi \lambda(\Delta)$, respectively. 


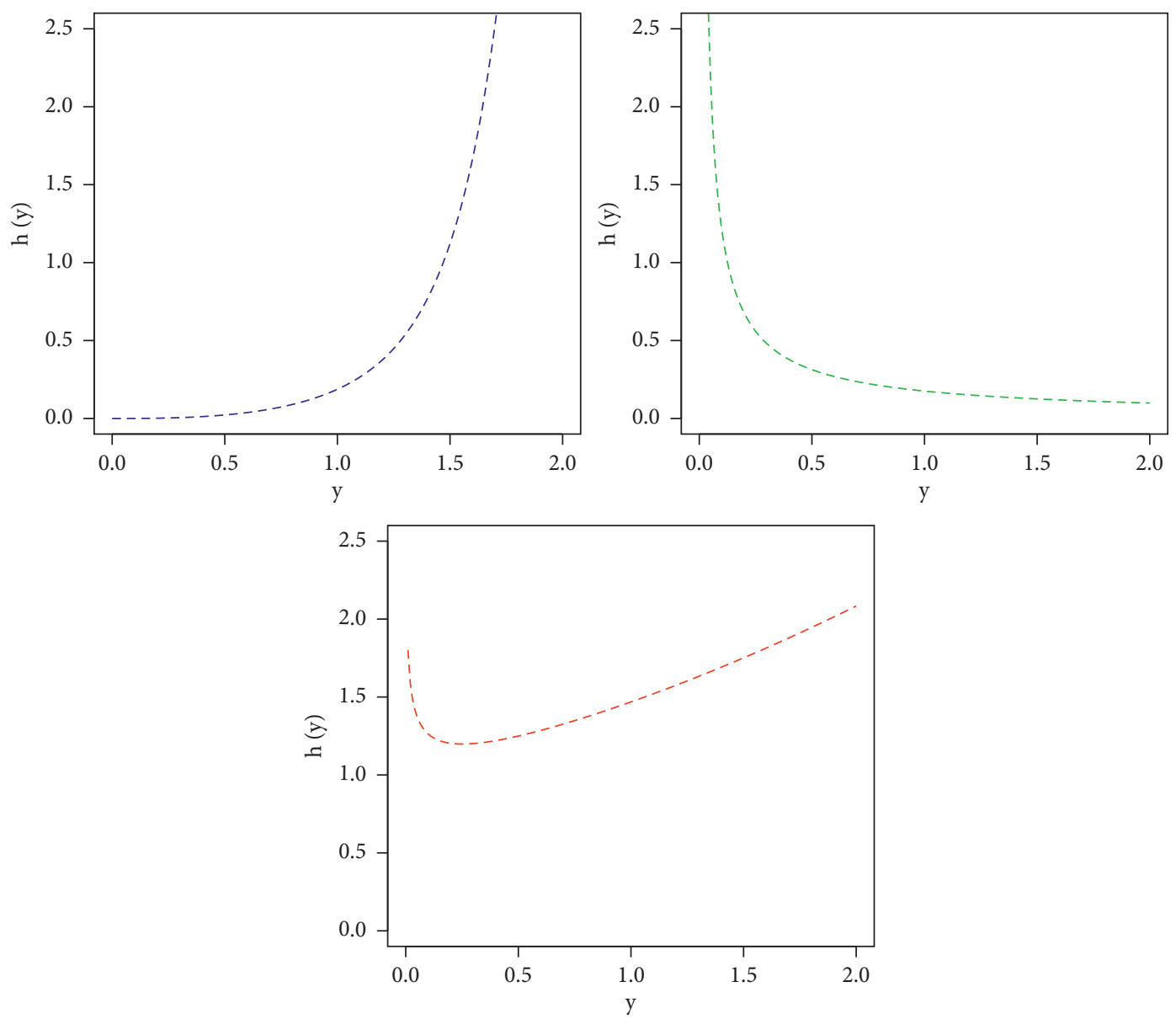

Figure 3: A visual illustration of $h(y ; \Delta)$ for different values of $\alpha, \varphi$, and $\phi$.

\section{Data Analyses}

Here, we show the applicability of the NAPow-Weibull model by analyzing two datasets. These datasets are taken from the sports sciences.

The first dataset consists of seventy-eight (78) observations and represents the waiting time duration till the first goal was scored in different football matches during 1964-2018. The first sports dataset is given by $14.00,14.00$, $13.00,13.00,12.00,12.00,12.00,12.00,12.00,12.00,12.00$, $11.00,11.00,11.00,10.80,10.69,10.12,10.00,10.00,10.00$, $10.00,9.90,9.60,9.55,9.00,9.00,9.00,9.00,8.70,8.30,8.10$, $8.10,8.00,8.00,8.00,8.00,8.00,8.00,8.00,7.80,7.70,7.69$, $7.66,7.42,7.30,7.27,7.22,7.00,7.00,7.00,7.00,7.00,7.00$, $6.00,6.00,6.00,6.00,6.00,6.00,6.00,5.00,5.00,4.00,4.00$, $4.00,3.90,3.69,3.60,3.57,3.55,3.17,3.00,3.00,2.80,2.80$, $2.56,2.20,2.10$.

The second dataset consists of seventy-six (78) observations and is also taken from the sports sciences. The second dataset also represents the waiting time duration till the first goal was scored in different football matches. The second dataset is given by $2.3520,2.4640,2.8672,3.1360$, $3.1360,3.3600,3.3600,3.5504,3.9760,3.9984,4.0320,4.1328$, $4.3680,4.4800,4.4800,4.4800,5.6000,5.6000,6.7200$, $6.7200,6.7200,6.7200,6.7200,6.7200,6.7200,7.8400$,
7.8400, 7.8400, 7.8400, 7.8400, 7.8400, 8.0864, 8.1424, 8.1760, $8.3104,8.5792,8.6128,8.6240,8.7360,8.9600,8.9600,8.9600$, $8.9600,8.9600,8.9600,8.9600,9.0720,9.0720,9.2960$, $9.7440,10.0800,10.0800,10.0800,10.0800,10.6960,10.7520$, $11.0880,11.2000,11.2000,11.2000,11.2000,11.3344$, $11.9728,12.0960,12.3200,12.3200,12.3200,13.4400$, $13.4400,13.4400,13.4400,13.4400,13.4400,13.4400$, 14.5600, 14.5600 .

The complete information about these datasets can retrieved at https://en.wikipedia.org/wiki/Fastest_goals_in_ association_football.

We fit the NAPow-Weibull and three other models (competitive models) to these datasets. The SFs of the competitive models (i) Weibull (Wei), (ii) exponentiated Weibull (Exp-Wei), and (iii) Kumaraswamy Weibull (KuWei) are given by

$$
\begin{gathered}
F(y ; \varphi, \phi)=1-e^{\varphi y^{\phi}}, \\
y \geq 0, \varphi>0, \phi>0, \\
F\left(y ; \varphi, \phi, \lambda_{1}\right)=1-\left(1-e^{-\varphi y^{\phi}}\right)^{\lambda_{1}}, \\
y \geq 0, \varphi>0, \phi>0, \lambda_{1}>0,
\end{gathered}
$$



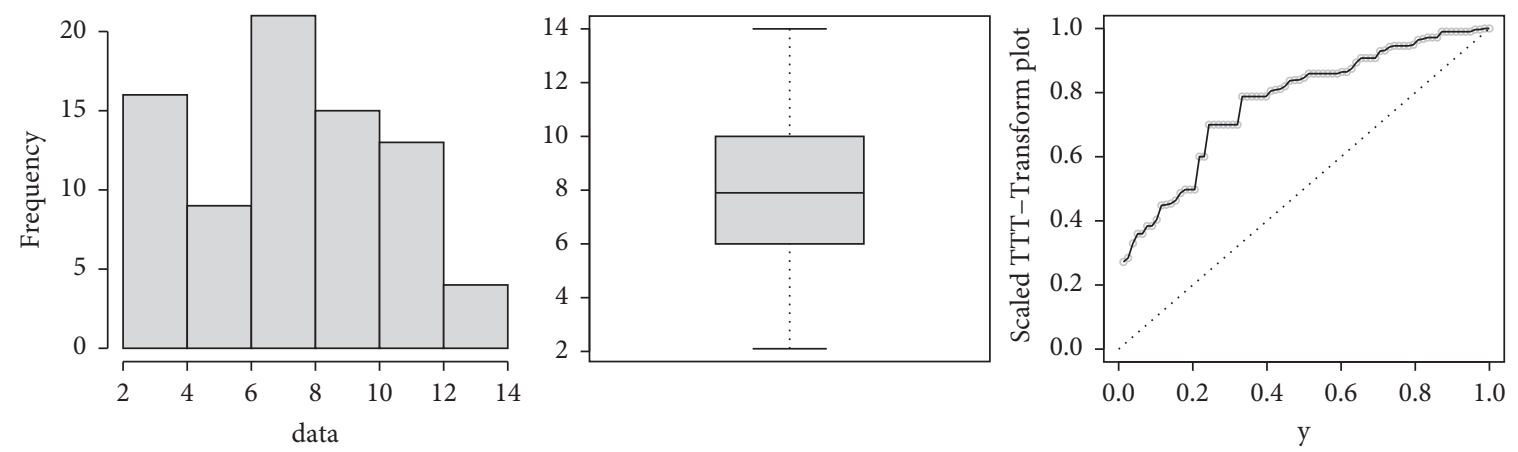

FIGURE 4: The graphs of BP, hist, and TTT corresponding to the first sports dataset.

TABLE 2: The values of $\widehat{\alpha}_{\mathrm{MLE}}, \widehat{\phi}_{\mathrm{MLE}}, \widehat{\varphi}_{\mathrm{MLE}}, \widehat{\lambda}_{1 \mathrm{MLE}}$, and $\widehat{\lambda}_{2 \mathrm{MLE}}$ using the first sports dataset.

\begin{tabular}{lccccc}
\hline Model & $\widehat{\alpha}_{\text {MLE }}$ & $\widehat{\phi}_{\text {MLE }}$ & $\widehat{\varphi}_{\text {MLE }}$ & $\widehat{\lambda}_{\text {MLE }}$ & $-\hat{\lambda}_{\text {2MLE }}$ \\
\hline NAPow-Weibull & 5.5958622 & 1.9570176 & 0.0053182 & - & - \\
Wei & - & 2.8122548 & 0.0023228 & - & - \\
Ex-Wei & - & 2.4055880 & 0.0064325 & 1.1416816 & 1.6756171 \\
Ku-Wei & - & 1.9164566 & 0.0075747 & 4.1447374 \\
\hline
\end{tabular}

Table 3: The IC measures of the NAPow-Weibull, Wei, Exp-Wei, and Ku-Wei models using the first sports dataset.

\begin{tabular}{lcccc}
\hline Model & AIC & CAIC & BIC & HQIC \\
\hline NAPow-Weibull & 397.9690 & 398.2933 & 405.0392 & 400.7993 \\
Wei & 398.9563 & 399.1163 & 406.6697 & 301.8431 \\
Exp-Wei & 400.1195 & 400.4439 & 407.1897 & 402.9498 \\
Ku-Wei & 437.1103 & 437.6582 & 446.5371 & 440.8840 \\
\hline
\end{tabular}

$$
\begin{aligned}
F\left(y ; \varphi, \phi, \lambda_{1}, \lambda_{2}\right) & =\left[1-\left(1-e^{-\varphi y^{\phi}}\right)^{\lambda_{1}}\right]^{\lambda_{2}}, \\
y & \geq 0, \varphi>0, \phi>0, \lambda_{1}>0, \lambda_{2}>0,
\end{aligned}
$$

respectively.

To figure out the flexibility/applicability of the fitted models (NAPow-Weibull, Wei, Exp-Wei, and Ku-Wei) using these two datasets, certain IC (information criteria) such as (i) AIC [23], (ii) BIC [24], (iii) CAIC [25], and (iv) HQIC [26] are considered. In addition to the IC, three g-o-f measures (goodness-of-fit measures) such as (i) Cramer-von Mises (CM) test statistic, (ii) Anderson-Darling (AD) test statistic, and (iii) Kolmogorov-Smirnov (KS) test statistic are also considered. Besides these seven statistical quantities, the $p$ value of the fitted models is also derived.

4.1. Data Analysis Using the First Dataset. In this section, we apply the NAPow-Weibull in comparison with the Wei, Exp-Wei, and Ku-Wei models to the first dataset taken from the sports sciences. The summary measures of the first sports dataset are given by minimum $=2.100,1$ st quartile $=6.000$, median $=7.900, \quad$ mean $=7.729, \quad 3 \mathrm{rd} \quad$ quartile $=10.000$, maximum $=14.000, \quad$ variance $=9.428, \quad$ standard deviation $=3.070$, and range $=11.900$. Corresponding to the first sports dataset, the plots of hist (histogram), PB (boxplot), and TTT (total time test) are provided in Figure 4.
In link to the first sports dataset, (i) the values of $\widehat{\alpha}_{M L E}, \widehat{\varphi}_{M L E}, \widehat{\phi}_{M L E}, \widehat{\lambda}_{1 M L E}$, and $\widehat{\lambda}_{2 M L E}$ of the NAPow-Weibull, Wei, Exp-Wei, and Ku-Wei models are obtained in Table 2, (ii) the values of the IC measures of the fitted models are reported in Table 3, and (iii) the values of the g-o-f measures are provided in Table 4.

Furthermore, for the first sports dataset, the (i) estimated DF, (ii) estimated SF, (iii) PP (probability-probability), and (iv) QQ (quantile-quantile) plots of the NAPow-Weibull model are sketched in Figure 5. Corresponding to the first sports dataset, the plots of the fitted DF and SF of the NAPow-Weibull model are obtained using the functions $W(y ; \widehat{\Phi})$ and $S(y ; \widehat{\Phi})$, respectively. The mathematical expressions of $W(y ; \widehat{\Phi})$ and $S(y ; \widehat{\Phi})$ are given by

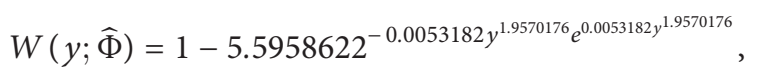

$$
\begin{aligned}
& y \geq 0 \text {, } \\
& S(y ; \widehat{\Phi})=5.5958622^{-0.0053182 y^{1.9570176} e^{0.0053182 y^{1.9570176}},} \\
& y>0
\end{aligned}
$$

respectively.

4.2. Data Analysis Using the Second Dataset. In this section, we again implement the NAPow-Weibull distribution in comparison with the Wei, Exp-Wei, and Ku-Wei models to the second sports dataset. The summary measures of the 
TABle 4: The g-o-f measures of the NAPow-Weibull, Wei, Exp-Wei, and Ku-Wei models using the first sports dataset.

\begin{tabular}{lcccc}
\hline Model & CM & AD & KS & $p$ value \\
\hline NAPow-Weibull & 0.081915 & 0.553162 & 0.087655 & 0.5867 \\
Wei & 0.103879 & 0.694736 & 0.104210 & 0.3653 \\
Ex-Wei & 0.122746 & 0.802127 & 0.133150 & 0.1259 \\
Ku-Wei & 0.130764 & 0.849605 & 0.117310 & 0.2333 \\
\hline
\end{tabular}
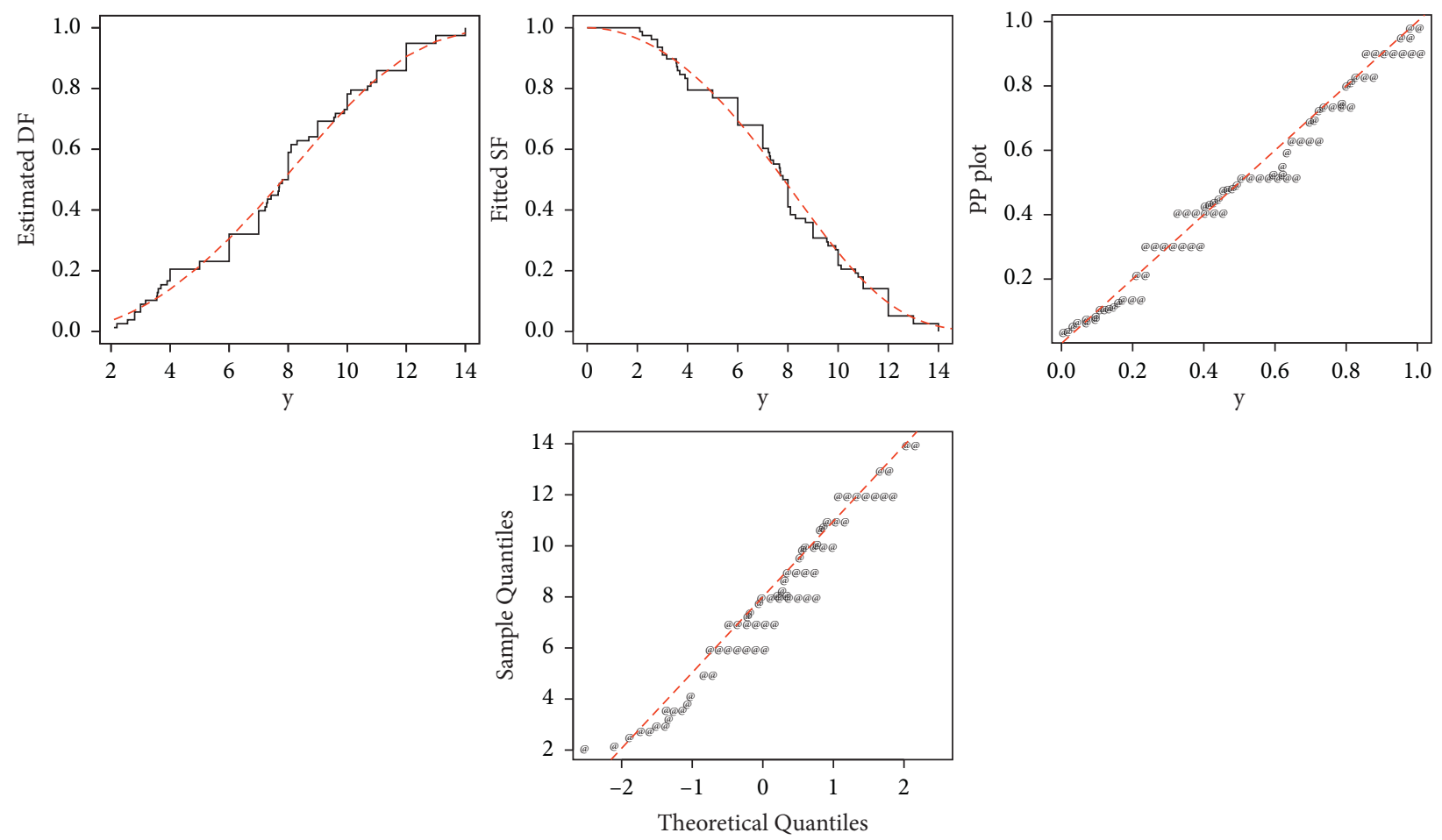

FIGURE 5: The estimated DF, SF, PP, and QQ plots of the NAPow-Weibull model corresponding to the first sports dataset.

second sports dataset are given by minimum $=2.352$, 1 st quartile $=6.720, \quad$ median $=8.680, \quad$ mean $=8.472, \quad 3 \mathrm{rd}$ quartile $=11.116, \quad$ maximum $=14.560, \quad$ variance $=10.792$, standard deviation $=3.285$, and range $=12.208$. Corresponding to the first sports data, the plots of hist, $\mathrm{PB}$, and TTT are presented in Figure 6.

Corresponding to the second sports dataset, (i) the values of $\widehat{\alpha}_{\mathrm{MLE}}, \widehat{\varphi}_{\mathrm{MLE}}, \widehat{\phi}_{\mathrm{MLE}}, \widehat{\lambda}_{1 \mathrm{MLE}}$, and $\widehat{\lambda}_{2 \mathrm{MLE}}$ of the NAPowWeibull, Wei, Exp-Wei, and Ku-Wei distributions are provided in Table 5, (ii) the values of the IC measures of the fitted models are presented in Table 6, and (iii) the values of the g-o-f measures are reported in Table 7.

In link to the second sports dataset, the (i) estimated DF, (ii) estimated SF, (iii) PP, and (iv) QQ plots of the NAPowWeibull model are presented in Figure 7. Corresponding to the second sports dataset, the plots of the fitted DF and SF of the NAPow-Weibull model are obtained via the expressions $W(y ; \widehat{\Phi})$ and $S(y ; \widehat{\Phi})$, respectively. For the second sports dataset, the mathematical expressions of $W(y ; \widehat{\Phi})$ and $S(y ; \widehat{\Phi})$ are, respectively, given by

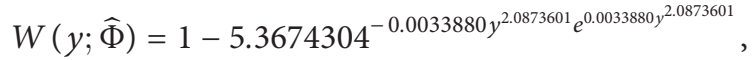

$$
\begin{aligned}
& y \geq 0 \text {, } \\
& S(y ; \widehat{\Phi})=5.3674304^{-0.0033880 y^{2.0873601} e^{0.0033880 y^{2.0873601}},} \\
& y>0 .
\end{aligned}
$$

4.3. Discussion. In this section, the NAPow-Weibull model was applied to two datasets taken from the sports sciences. The first dataset consists of seventy-eight observations and was taken from the sports sciences. The NAPow-Weibull, Wei, Exp-Wei, and $\mathrm{Ku}-\mathrm{Wei}$ models were applied to these data. Based on the IC measures, it is observed that the NAPow-Weibull was the best model. Furthermore, it is also observed that the Wei model was the second-best competitor in terms of the IC measures, whereas the Exp-Weibull model was observed to be the second-best model based on the $p$ value. 

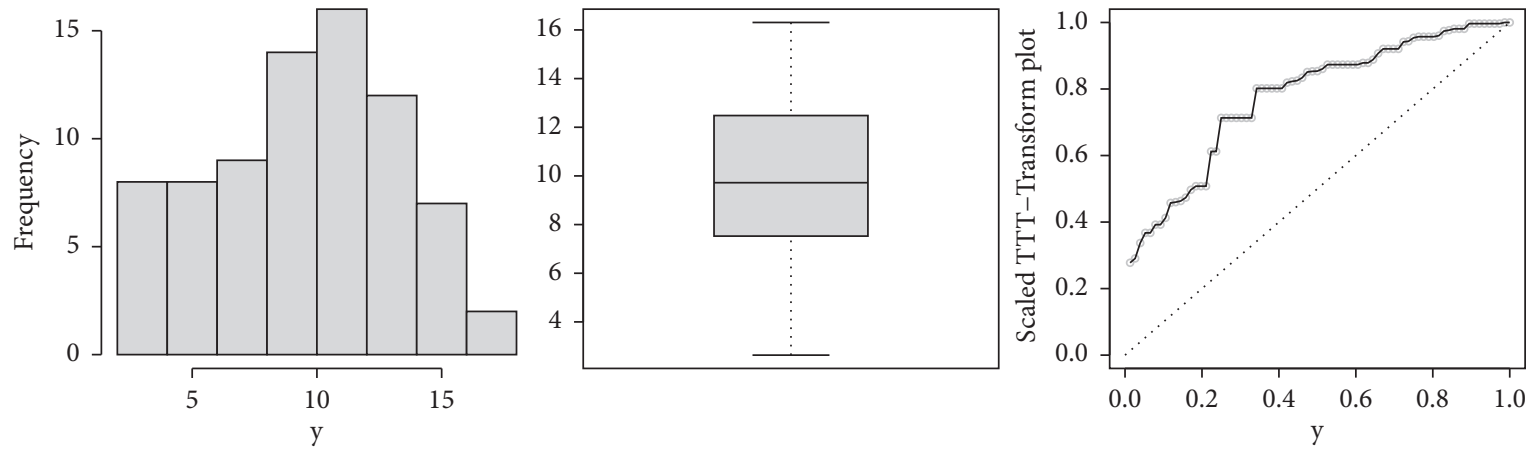

Figure 6: The plots of BP, hist, and TTT corresponding to the second sports dataset.

TABLE 5: The values of $\widehat{\alpha}_{\mathrm{MLE}}, \widehat{\phi}_{\mathrm{MLE}}, \widehat{\varphi}_{\mathrm{MLE}}, \widehat{\lambda}_{1 \mathrm{MLE}}$, and $\widehat{\lambda}_{2 \mathrm{MLE}}$ using the second sports dataset.

\begin{tabular}{|c|c|c|c|c|c|}
\hline Model & $\widehat{\alpha}_{\mathrm{MLE}}$ & $\widehat{\phi}_{\mathrm{MLE}}$ & $\widehat{\varphi}_{\text {MLE }}$ & $\widehat{\lambda}_{1 \mathrm{MLE}}$ & $\widehat{\lambda}_{2 \mathrm{MLE}}$ \\
\hline NAPow-Weibull & 5.3674304 & 2.0873601 & 0.0033880 & - & - \\
\hline Wei & - & 2.7001948 & 0.0023373 & - & - \\
\hline Ex-Wei & - & 2.1687800 & 0.0106778 & 1.5569487 & - \\
\hline Ku-Wei & - & 1.1827705 & 0.0898311 & 3.8295433 & 3.1540636 \\
\hline
\end{tabular}

Table 6: The IC measures of the NAPow-Weibull, Wei, Exp-Wei, and Ku-Wei models using the second sports dataset.

\begin{tabular}{lcccc}
\hline Model & AIC & CAIC & BIC & HQIC \\
\hline NAPow-Weibull & 396.5795 & 396.9128 & 403.5717 & 399.3739 \\
Wei & 397.8422 & 398.0066 & 402.5037 & 399.7052 \\
Exp-Wei & 401.9609 & 402.2943 & 408.9531 & 404.7554 \\
Ku-Wei & 407.2465 & 407.8099 & 416.5695 & 410.9724 \\
\hline
\end{tabular}

TABle 7: The g-o-f measures of the NAPow-Weibull, Wei, Exp-Wei, and Ku-Wei models using the second sports dataset.

\begin{tabular}{lcccc}
\hline Model & CM & AD & KS & $p$ value \\
\hline NAPow-Weibull & 0.085418 & 0.618361 & 0.086839 & 0.6153 \\
Wei & 0.129871 & 0.872488 & 0.126340 & 0.1766 \\
Ex-Wei & 0.170103 & 1.092799 & 0.128460 & 0.1627 \\
Ku-Wei & 0.223717 & 1.383692 & 0.141980 & 0.0933 \\
\hline
\end{tabular}
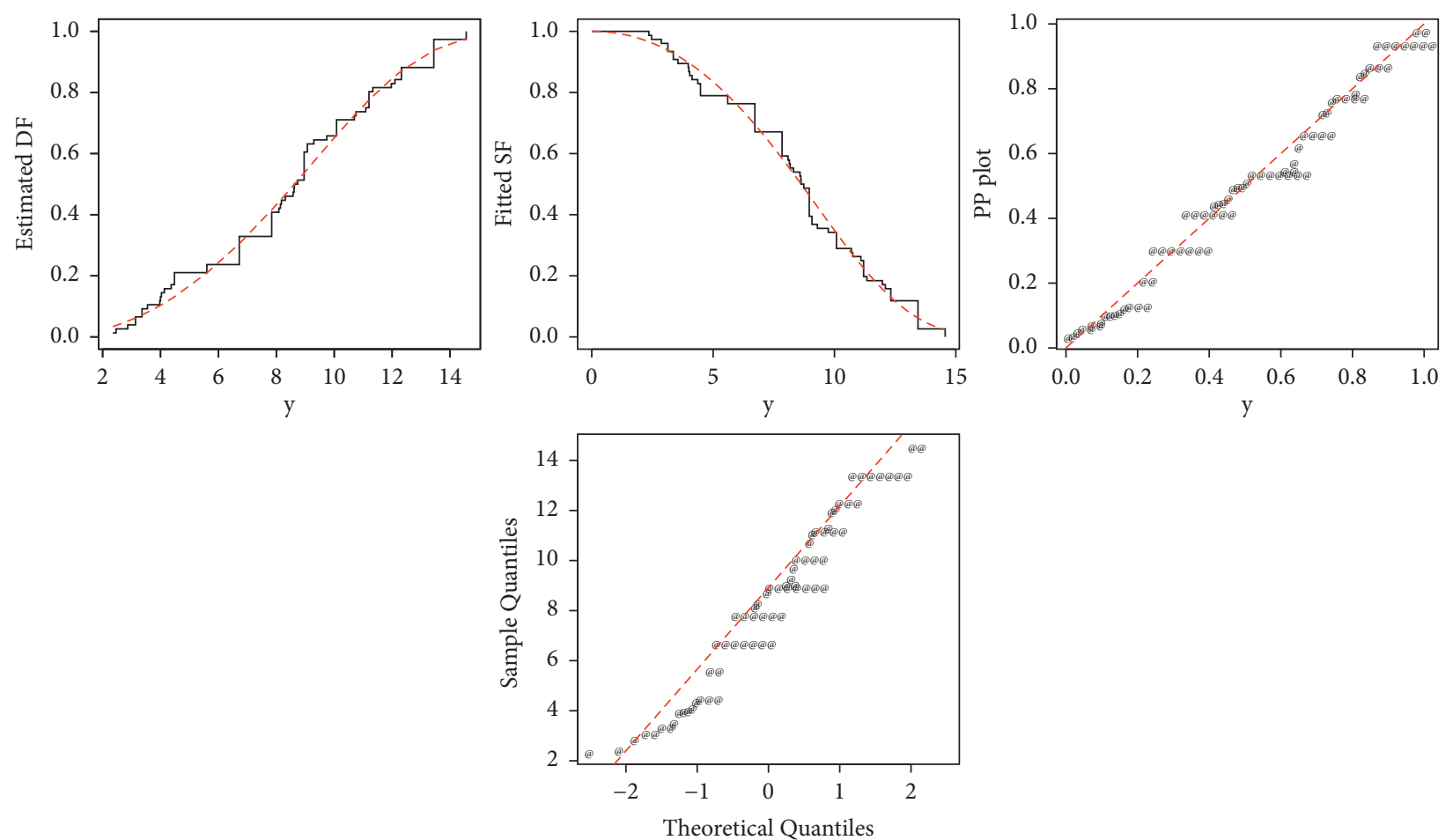

FIgURE 7: The estimated DF, SF, PP, and QQ plots of the NAPow-Weibull model corresponding to the second sports dataset. 
The second dataset consists of seventy-six observations and was also taken from the sports sciences. Again, the NAPow-Weibull, Wei, Exp-Wei, and Ku-Wei models were applied to the second sports dataset. Based on the IC and g-o-f measures, it is again observed that the NAPow-Weibull was the best competitor than the Wei, Exp-Wei, and Ku-Wei distributions.

\section{Final Remarks}

In this article, a new family, that can be used as an alternative to a NWei- $X$ family, is introduced. Numerous distributional properties of the new family are obtained. There are several advantages of using the proposed family. First, a very simple approach was proposed to modify the existing distributions by adding an extra parameter. Second, the proposed method can be useful to introduce new distributions belonging to $T$ $X$ family. Third, the existing distributions having a closedform of DF were extended. Furthermore, a special model (NAPow-Weibull) of the new alpha power family was studied. Some graphical behaviors of the PDF and HF of the NAPow-Weibull model were obtained. Finally, two applications were discussed to illustrate the NAPow-Weibull distribution. Based on the findings using these two datasets, it is shown that the NAPow-Weibull model was an appropriate model for dealing with the data in sports and other related sciences.

\section{Data Availability}

The data used to support the findings of this study are included within the article.

\section{Conflicts of Interest}

The authors declare that they have no conflicts of interest.

\section{Authors' Contributions}

Gao Shengjie and Alisa Craig were responsible for conceptualization and methodology. Gao Shengjie supervised the study. Gao Shengjie, Alisa Craig, and Getachew Tekle Mekiso were responsible for original draft preparation and review and editing. Alisa Craig and Getachew Tekle Mekiso were responsible for formal analysis and software.

\section{References}

[1] A. S. Malehi, F. Pourmotahari, and K. A. Angali, "Statistical models for the analysis of skewed healthcare cost data: a simulation study," Health economics review, vol. 5, no. 1, pp. 11-16, 2015.

[2] E. Kabir, S. Guikema, and B. Kane, "Statistical modeling of tree failures during storms," Reliability Engineering \& System Safety, vol. 177, pp. 68-79, 2018.

[3] R. Pierce, H. Chick, J. Watson, M. Les, and M. Dalton, “A statistical literacy hierarchy for interpreting educational system data," Australian Journal of Education, vol. 58, no. 2, pp. 195-217, 2014.

[4] H. E. Andersen, B. Kronvang, S. E. Larsen, C. C. Hoffmann, T. S. Jensen, and E. K. Rasmussen, "Climate-change impacts on hydrology and nutrients in a Danish lowland river basin," The Science of the Total Environment, vol. 365, no. 1-3, pp. 223-237, 2006.

[5] M. Leo, S. Sharma, and K. Maddulety, "Machine learning in banking risk management: a literature review," Risks, vol. 7, no. 1, p. 29, 2019.

[6] N. Senin, S. Catalucci, M. Moretti, and R. K. Leach, "Statistical point cloud model to investigate measurement uncertainty in coordinate metrology," Precision Engineering, vol. 70, pp. 44-62, 2021.

[7] S. Ullah, T. J. Gabbett, and C. F. Finch, "Statistical modelling for recurrent events: an application to sports injuries," British Journal of Sports Medicine, vol. 48, no. 17, pp. 1287-1293, 2014.

[8] Z. Ahmad, G. G. Hamedani, and N. S. Butt, "Recent developments in distribution theory: a brief survey and some new generalized classes of distributions," Pakistan Journal of Statistics and Operation Research, vol. 15, no. 1, pp. 87-110, 2019.

[9] A. Alzaatreh, C. Lee, and F. Famoye, "A new method for generating families of continuous distributions," Metron, vol. 71, no. 1, pp. 63-79, 2013.

[10] C. Alexander, G. M. Cordeiro, E. M. M. Ortega, and J. M. Sarabia, "Generalized beta-generated distributions," Computational Statistics \& Data Analysis, vol. 56, no. 6, pp. 1880-1897, 2012.

[11] M. M. Ristić and N. Balakrishnan, "The gamma-exponentiated exponential distribution," Journal of Statistical Computation and Simulation, vol. 82, no. 8, pp. 1191-1206, 2012.

[12] K. Zografos and N. Balakrishnan, "On families of beta- and generalized gamma-generated distributions and associated inference," Statistical Methodology, vol. 6, no. 4, pp. 344-362, 2009.

[13] H. Torabi and N. Montazeri Hedesh, "The gamma-uniform distribution and its applications," Kybernetika, vol. 48, no. 1, pp. 16-30, 2012.

[14] A. Alzaghal, F. Famoye, and C. Lee, "Exponentiated TX family of distributions with some applications," International Journal of Statistics and Probability, vol. 2, no. 3, p. 31, 2013.

[15] Z. Ahmad, E. Mahmoudi, S. Dey, and S. K. Khosa, "Modeling vehicle insurance loss data using a new member of T-X family of distributions," Journal of Statistical Theory and Applications, vol. 19, no. 2, pp. 133-147, 2020.

[16] Z. Ahmad, E. Mahmoudi, and O. Kharazmi, "On modeling the earthquake insurance data via a new member of the T-X family," Computational Intelligence and Neuroscience, vol. 2020, Article ID 7631495, 20 pages, 2020.

[17] Z. Ahmad, E. Mahmoudi, R. Roozegar, M. Alizadeh, and A. Z. Afify, "A new exponential-X family: modeling extreme value data in the finance sector," Mathematical Problems in Engineering, vol. 2021, Article ID 8759055, 14 pages, 2021.

[18] Z. Ahmad, E. Mahmoudi, M. Alizadeh, R. Roozegar, and A. Z. Afify, "The exponential TX family of distributions: properties and an application to insurance data," Journal of Mathematics, vol. 2021, Article ID 3058170, 18 pages, 2021.

[19] Z. Ahmad, M. Elgarhy, and G. G. Hamedani, "A new Weibull$\mathrm{X}$ family of distributions: properties, characterizations and applications," Journal of Statistical Distributions and Applications, vol. 5, no. 1, pp. 1-18, 2018.

[20] H. Torabi and N. H. Montazeri, "The logistic-uniform distribution and its applications," Communications in Statistics Simulation and Computation, vol. 43, no. 10, pp. 2551-2569, 2014. 
[21] M. H. Tahir, G. M. Cordeiro, A. Alzaatreh, M. Mansoor, and M. Zubair, "The logistic-X family of distributions and its applications," Communications in Statistics - Theory and Methods, vol. 45, no. 24, pp. 7326-7349, 2016.

[22] Q. Liao, Z. Ahmad, E. Mahmoudi, and G. G. Hamedani, "A new flexible bathtub-shaped modification of the Weibull model: properties and applications," Mathematical Problems in Engineering, vol. 2020, Article ID 3206257, 11 pages, 2020.

[23] H. Akaike, "A new look at the statistical model identification," In Selected Papers of Hirotugu Akaike, Springer, New York, NY, pp. 215-222, 1974.

[24] G. Schwarz, "Estimating the dimension of a model," Annals of Statistics, vol. 6, no. 2, pp. 461-464, 1978.

[25] H. Bozdogan, "Model selection and Akaike's Information Criterion (AIC): the general theory and its analytical extensions," Psychometrika, vol. 52, no. 3, pp. 345-370, 1987.

[26] E. J. Hannan and B. G. Quinn, "The determination of the order of an autoregression," Journal of the Royal Statistical Society: Series B, vol. 41, no. 2, pp. 190-195, 1979. 